\title{
Glucocorticoids cause VE-cadherin upregulation and cytoskeletal rearrangements in the blood-brain barrier endothelial cEND cell
}

line

\author{
KG Blecharz*1, D Drenckhahn² and C Förster ${ }^{1}$
}

\begin{abstract}
Address: ${ }^{1}$ University of Würzburg, Zentrum für operative Medizin, Experimentelle Anästhesiologie, Würzburg, Germany and ${ }^{2}$ University of WürzburgInstitut für Anatomie und Zellbiologie II, Universität Würzburg, Würzburg, Germany

* Corresponding author
\end{abstract}

from 12th Joint Meeting of the Signal Transduction Society (STS). Signal Transduction: Receptors, Mediators and Genes

Weimar, Germany. 29-31 October 2008

Published: 26 February 2009

Cell Communication and Signaling 2009, 7(Suppl I):A95 doi:I0.1।86/1478-8IIX-7-SI-A95

This abstract is available from: http://www.biosignaling.com/content/7/SI/A95

(c) 2009 Blecharz et al; licensee BioMed Central Ltd.

The demonstration that glucocorticoid-mediated activation can control occludin expression in brain capillary endothelial cells has provided a new avenue of research in the field of blood brain-barrier (BBB) permeability regulation. In order to identify more key genes involved in glucocorticoid-mediated regulation of BBB permeability, we used CDNA microarrays to study changes in gene expression in dexamethasone-treated brain capillary endothelial cEND cells: Primarily, we observed changes in expression of the VE-cadherin gene involved in cell adhesion. Vascular-endothelial-cadherin (VE-cadherin) is an endothelial cell-specific adhesion protein localized in cell-cell contacts. It is known as an important determinant of vascular architecture and endothelial cell survival. Quantitative real-time PCR showed an upregulation of VE-cadherin expression exclusively in cEND cells, in accordance to previous observations made for the occludin gene. Subsequently, we verified divergent transcriptional activation of the VE-cadherin gene by dexamethasone. Furthermore, we measured the change in protein levels of VE-cadherin and demonstrated a transactivation of the VE-cadherin promoter in cEND cells via dexamethasone. Dexamethasone was further shown to induce cellular differentiation into a cobblestone cellular morphology and reinforcement of adherens junctions concomitant with the increased anchorage of VE-cadherin to the actin cytoskeleton. We thus propose that glucocorticoid effects on VE-cadherin protein synthesis and organization are important for the formation of both adherens and tight junction, and for improved barrier properties in microvascular brain endothelial cells.

To find cell- or tissue-specific ligands that could be used in therapeutic regime of barrier disorder diseases it is necessary to study and understand the molecular mechanisms of beneficial effects of glucocorticoid action on BBB-genes involved in BBB-permeability regulation. 